\title{
Phase plane method for the parameter identification of strongly nonlinear systems
}

\author{
Tang Jiashi \\ Department of Engineering Mechanics, Hunan \\ University, Changsha 410082, P.R.China
}

A phase plane method is presented for the parameter identification of strongly nonlinear systems. The phase trajectory equation is taken as the mathematical model and the system parameters are identified by using the optimum technique. The method can be applied to conservative and nonconservative systems. The value ranges of the parameters are not limited. All examples simulated by computer show that the efficiency and accuracy of this approach are very good.

\section{Introduction}

Finding approximate solutions of strongly nonlinear systems is a problem which mechanics researchers are concerned about. Though there are effective methods for the analysis of some strongly nonlinear vibrations [1-3], the study of strongly nonlinear systems is not so well developed as weakly nonlinear systems because of the difficulty in the mathematical theory. Since finding analytical solutions is difficult, few study is carried on the parameter identification of strongly nonlinear systems. Two methods of the parameter identification of weakly nonlinear systems in time and frequency domain have been presented in the references [4,5]. The prerequisite of the two methods is to obtain the approximate solution of the nonlinear vibration system, so they are also applicable to the strongly nonlinear systems which have analytical solution. Nayfeh [6] have studied parametric identification of nonlinear systems. Yasuda et al. [7,8] have proposed the new experimental identification applicable to elastic structures.

Though the analytical solutions of some strongly nonlinear systems can not be determined, the phase trajectory equations can be established. The mathematical mode for parameter identification is provided. By using the optimum method, we fit the level curve in the phase plane and identify the parameters of strongly nonlinear systems. The method can be applied to conservative and non-conservative systems.

We consider the autonomous system governed by the following equation.

$$
\ddot{x}+g(x, \dot{x})=0
$$

where $g(x, \dot{x}) \in C^{1}\left(R^{2}, R\right)$. If $\alpha, \beta, \ldots$ are the coefficients of the terms in $g(x, \dot{x})$, Eq. (1) can be rewritten in the form

$$
\ddot{x}+g(x, \dot{x}, \alpha, \beta, \ldots)=0
$$

Let $\dot{x}=y$, Eq. (2) becomes

$$
\left\{\begin{array}{l}
\dot{x}=y \\
\dot{y}=-g(x, y, \alpha, \beta, \ldots)
\end{array}\right.
$$

The initial conditions of Eq. (3) are $x(0)=$ $x_{0}, y(0)=y_{0}$. Let $\mathcal{D}$ be a region in the phase plane $(x, y),(x, y) \in R^{2}$, and assume that every $\left(x_{0}, y_{0}\right) \in \mathcal{D}$. According to the continuous theorem the initial value should satisfy the following set of equations

$$
\left\{\begin{array}{l}
x=x\left(x_{0}, y_{0}\right) \\
y=y\left(x_{0}, y_{0}\right)
\end{array}\right.
$$

where $\left(x_{0}, y_{0}\right) \in \mathcal{D}$ is continuous, and Eq. (4) defines a continuous map

$$
T:\left(x_{0}, y_{0}\right) \mid \rightarrow(x, y)
$$

or

$$
T: \mathcal{D} \rightarrow R^{2}
$$

Here $\left(x_{0}, y_{0}\right)$ is a fixed point of the map $T$ : $T\left(x_{0}, y_{0}\right)=\left(x_{0}, y_{0}\right)$. If the initial values $\left(x_{0}, y_{0}\right)$ are given, a definite and continuous curve (4) can be determined theoretically. We take Eq. (4) as the mathematical model for parameter identification. However, finding the analytical solution of Eq. (3) is quite difficult.

Equation (3) is written as

$$
\frac{d y}{d x}=-\frac{g(x, y, \alpha, \beta, \ldots)}{y}
$$

we establish the phase trajectory equation of some strongly nonlinear systems in the form 


$$
f(x, y, \alpha, \beta, \ldots)=0
$$

This phase trajectory may be or may not be a close curve. No matter which type the phase trajectory is, Eq. (6) is regarded as the mathematical model for parameter identification. The level curve is determined by fitting it with the measured date $\left\{\tilde{x}_{i}, \tilde{y}_{i}\right\}(i=1,2, \ldots, n)$, thus parameters of the nonlinear system are identified.

As the errors are not avoided in the measurement, each pair $\left\{\tilde{x}_{i}, \tilde{y}_{i}\right\}$ may not satisfy Eq. (6) exactly. There are deviations between the observed data and the regression equation values. The leastsquare method will give a curve best close best close to the true phase trajectory, thereby we find the parameters $\alpha, \beta, \ldots$ of the nonlinear systems. The value ranges of the parameters $\alpha, \beta, \ldots$ are not limited.

This is a problem of optimization as follows

$$
\begin{aligned}
S(\alpha, \beta, \ldots) & =\sum_{i=1}^{n}\left[f\left(\tilde{x}_{i}, \tilde{y}_{i}, \alpha, \beta, \ldots\right)\right]^{2} \\
& =\min
\end{aligned}
$$

where $S$ is the objective function and $\alpha, \beta, \ldots$ are the design variables which are calculated by using the optimum principle. When the objective fuction $S(\alpha, \beta, \ldots)$ has more than one minimum the parameters of the system are determined by the experiment and analysis.

\section{Parameter identification of strongly nonlinear system governed by $\ddot{x}+g(x)=0$}

We consider a conservative system governed by the following equation

$$
\ddot{x}+g(x)=0
$$

where $g(x) \in C^{1}(R, R)$. In mathematical analysis Eq. (8) will be strongly nonlinear if function $g(x)$ satisfies

$$
\lim _{|x| \rightarrow \infty} \frac{g(x)}{x}=\infty
$$

In mechanics the behavior of a system depends on the coefficients of nonlinear terms. If the value of every coefficient is far less than 1 , the equation will be weakly nonlinear; if it is not, the equation will be strongly nonlinear.

In phase plane Eq. (8) is written as

$$
\frac{d y}{d x}=-\frac{g(x)}{y}
$$

or

$$
\frac{1}{2} y^{2}=-\int_{0}^{x} g(x) d x+C
$$

thus the first integration for Eq. (9) is

$$
\frac{1}{2} y^{2}+G(x)=C
$$

where $G(x)$ is a potential function.

If $g(x)$ is an odd polynomial, $G(x)$ will be an even polynomial. For any constant $C=C_{0}>0$ Eq. (10) determines a close trajectory $\Gamma_{C O}$ of Eq. (9). It intersects $\mathrm{x}$-axis at two points $\left(-x_{0}, 0\right.$ and $\left(x_{0}, 0\right)$, where $\pm x_{0}$ satisty $G\left( \pm x_{0}\right)=C_{0}$. The phase diagram determined by Eq. (10) is a bunch of close trajectories whose center $\mathrm{O}$ is the origin of coordinates. If $g(x)$ is an arbitrary polynomial, Eq. (10) will always be analytic and its phase trajectory is symmetric with rspect to $\mathrm{x}$-axis. Thus we can identify parameters of nonlinear system using the phase trajectory equation.

Example 1. Consider a nonlinear vibration system governed by Duffing's equation of the form

$$
\ddot{x}+\alpha x+\beta x^{3}=0
$$

Its phase trajectory equation is

$$
y^{2}=C-2 \int_{0}^{x}\left(\alpha x+\beta x^{3}\right) d x
$$

or

$$
y^{2}=C-\alpha x^{2}-\frac{1}{2} \beta x^{4}
$$

The initial conditions of Eq. (11) are $x(0)=x_{0}$ and $y(0)=y_{0}$. Introducing the initial conditions into Eq. (12) leads to

$$
C=y_{0}^{2}+\alpha x_{0}^{2}+\frac{1}{2} \beta x_{0}^{4}
$$

Equation (12) is rewritten in the form

$$
y^{2}+\alpha x^{2}+\frac{1}{2} \beta x^{4}-y_{0}^{2}-\alpha x_{0}^{2}-\frac{1}{2} \beta x_{0}^{4}=0
$$

The problem of optimization for identifying parameters $\alpha, \beta$ is

$$
\begin{aligned}
& S(\alpha, \beta)=\sum_{i=1}^{n} \\
& \left(\tilde{y}_{i}^{2}+\alpha \tilde{x}_{0}^{2}+\frac{1}{2} \beta \tilde{x}_{i}^{4}-y_{0}^{2}-d x_{0}^{2}-\frac{1}{2} \beta x_{0}^{4}\right)^{2} \\
& =\min
\end{aligned}
$$

Example 2. Consider a physical pendulum as shown is Fig. 1. Its differential equations of motion is 


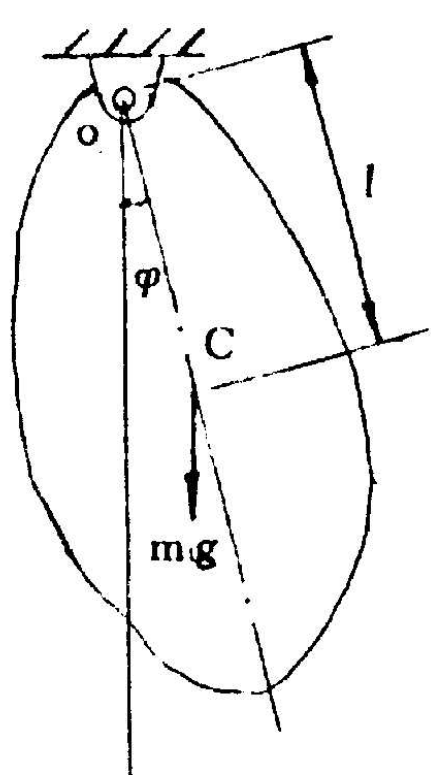

Fig. 1. Physical pendulum.

$$
\ddot{\varphi}+\frac{m g l}{J} \sin \varphi=0
$$

where $m$ is the mass of the pendulum, $J$ is the moment of inertia about the horizontal asix, $g$ is the acceleration of gravity, $l$ is the distance between the hinge $\mathrm{O}$ and the center of mass $C$. let $d=\frac{m g l}{J}$, the Eq. (15) becomes

$$
\ddot{\varphi}+\alpha \sin \varphi=0
$$

For large amplitude $\sin \varphi \approx \varphi$ is invalid. Let $\Psi=\dot{\varphi}$, and we find the phase trajectory equation in the form

$$
\varphi^{2}=C+2 \alpha \cos \varphi
$$

Measuring a set of angles of rotation and angular velocities of the pendulum and using Eq. (17) we identify the parameter $a$ and evaluate the moment of inertia of the pendulum with a higher precision.

\section{Parameter identification of strongly nonlinear systems governed by $\ddot{x}+g(x, \dot{x})=0$}

Consider the nonconservative system governed by the following equation

$$
\ddot{x}+g(x, \dot{x})=0
$$

In phase plane Eq. (18) is written as

$$
\frac{d y}{d x}=-\frac{g(x, y)}{y}
$$

or

$$
\frac{d y^{2}}{d x}=-2 g(x, y)
$$

If the first order differential Eq. (19) or (20) is expressed in the following form:

(a) separation of variables

$$
\frac{d y}{d x}=X(x) Y(y)
$$

or

$$
\frac{d y^{2}}{d x}=X(x) Y\left(y^{2}\right)
$$

(b) first order form (include the quasi-first order form)

$$
\frac{d y}{d x}+P(x) y=Q(x)
$$

or

$$
\frac{d y^{2}}{d x}+P(x) y^{2}=Q(x)
$$

(c) form

$$
\frac{d y}{d x}=X_{0}(x)+X_{1}(x) y+X_{2}(x) y^{2}
$$

we can obtain the phase trajectory equation for parameter identification.

Example 3. Consider equation

$$
\ddot{x}+\alpha x+\beta x \dot{x}^{2}=0
$$


Table 1

Numerical solutions of equation

\begin{tabular}{cccccccc}
\hline$i$ & 0 & 1 & 2 & 3 & 4 & 5 & 6 \\
$t$ & 0 & 0.2 & 0.4 & 6 & 0.8 & 1.0 & 1.2 \\
$\tilde{x}_{i}$ & 1 & 0.9798 & 0.9167 & 0.8025 & 0.6225 & 0.3593 & 0.0208 \\
$\tilde{y}_{i}$ & 0 & -0.2041 & -0.4338 & -0.7204 & -1.0964 & -1.5342 & -1.7865 \\
\hline & 7 & 8 & 9 & 10 & 11 & 12 & 13 \\
& 1.4 & 1.6 & 1.8 & 2.0 & 2.2 & 2.4 & 2.6 \\
& -0.3230 & -0.5964 & -0.7853 & -0.9062 & -0.9748 & -0.9997 & -0.9843 \\
-1.5807 & -1.1463 & -0.7591 & -0.4635 & -0.2289 & -0.0233 & 0.1795 \\
\hline & 14 & 15 & 16 & 17 & 18 & 19 & 20 \\
& 2.8 & 3.0 & 3.2 & 3.4 & 3.6 & 3.8 & 4.0 \\
& -0.9264 & -0.8188 & -0.6474 & -0.3944 & -0.0623 & 0.2857 & 0.5691 \\
0.4048 & 0.6830 & 1.0477 & 1.4485 & 1.7793 & 1.6242 & 1.1971 \\
\hline & 21 & 22 & 23 & 24 & & & \\
& 4.2 & 4.4 & 4.6 & 4.8 & & & \\
& 0.7672 & 0.8951 & 0.9691 & 0.9989 & & & \\
\hline & 0.7891 & 0.4941 & 0.2542 & 0.0466 & & & \\
\hline
\end{tabular}

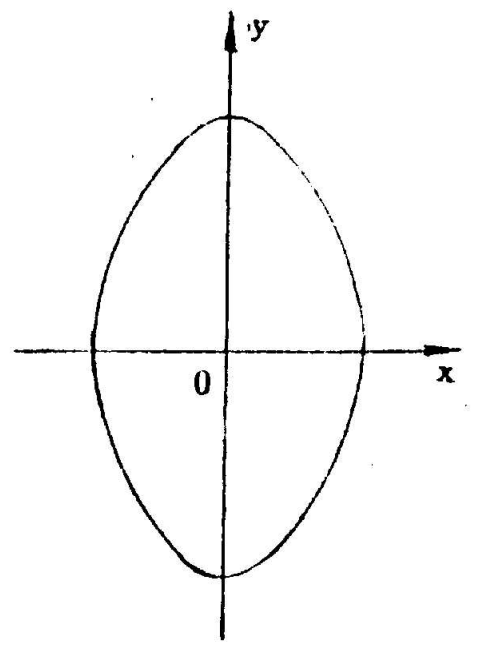

Fig. 2. Phase curve of equation $\ddot{x}+x+2 x \dot{x}^{2}=0$.

Let $y=\dot{x}$, and in phase plane the differential Eq. (26) is written as

$$
\frac{d y}{d x}=\frac{-\alpha x-\beta x y^{2}}{y}
$$

or

$$
\frac{d y^{2}}{d x}+2 \beta x y^{2}=-2 \alpha x
$$

We have

$$
y^{2}=e^{-\int 2 \beta x d x}\left(\int-2 \alpha x e^{\int 2 \beta x d x} d x+C\right)
$$

Completing the integration, we obtain

$$
y^{2}=C e^{-\beta x^{2}}-\frac{\alpha}{\beta}
$$

The constant $C$ of integration is determined by introducing $x(0)=x_{0}, y(0)=y_{0}$, and the result is

$$
C=\left(y_{0}^{2}+\frac{\alpha}{\beta}\right) e^{\beta x_{0}^{2}}
$$

The phase trajectory equation is

$$
y^{2}-\left(y_{0}^{2}+\frac{\alpha}{\beta}\right) e^{\beta\left(x_{0}^{2}-x^{2}\right)}+\frac{\alpha}{\beta}=0
$$

Thus, the parameter identification of the system governed by Eq. (26) is defined by

$$
\begin{aligned}
& S(\alpha, \beta) \\
= & \sum_{i=1}^{n}\left[\tilde{y}_{0}^{2}-\left(y_{0}^{2}+\frac{\alpha}{\beta}\right) e^{\beta\left(x_{0}^{2}-\tilde{x}_{i}^{2}\right)}+\frac{\alpha}{\beta}\right]^{2}
\end{aligned}
$$




$$
=\min
$$

Let $\alpha=1, \beta=2, x_{0}=1, y_{0}=0$ and Eq. (26) becomes

$$
\ddot{x}+x+2 x \dot{x}^{2}=0
$$

The numerical solution $\left\{\tilde{x}_{i}, \tilde{y}_{i}\right\} \quad(i=1,2, \ldots, 24)$ are given in Talbe 1 . The phase trajectory is shown in Fig. 2.

Adopt $\left\{\tilde{x}_{i}, \tilde{y}_{i}\right\}$ in Table 1 as the tested data to identify the parameters and of the nonlinear system (26), that is to find an optimum solution to the objective function (31). The result is

$$
\alpha=1.0052, \quad \beta=1.9917
$$

Example 4. Consider equation

$$
\ddot{x}+\alpha x+\beta x^{3}+\gamma \dot{x}^{2}=0
$$

Its phase trajectory equation is

$$
\frac{d y}{d x}=\frac{-\alpha x-\beta x^{3}-\gamma y^{2}}{y}
$$

or

$$
\frac{d y^{2}}{d x}+2 \gamma y^{2}=-2 \alpha x-2 \beta x^{3}
$$

The result of integration is

$$
\begin{aligned}
y^{2}= & C e^{-2 \gamma x}-\frac{\beta}{\gamma} x^{3}+\frac{3 \beta}{2 \gamma^{2}} x^{2} \\
& -\frac{2 \alpha \gamma^{2}+3 \beta}{2 \gamma^{3}} x+\frac{2 \alpha \gamma^{2}+3 \beta}{4 \gamma^{4}} x
\end{aligned}
$$

The phase trajectory defined by Eq. (33) is shown in Fig. 3. In phase plane it has a boundary

$$
\begin{aligned}
y^{2}= & -\frac{\beta}{\gamma} x^{3}+\frac{3 \beta}{2 \gamma^{2}} x^{2}-\frac{2 \alpha \gamma^{2}+3 \beta}{2 \gamma^{3}} x \\
& +\frac{2 \alpha \gamma^{2}+3 \beta}{4 \gamma^{4}} x
\end{aligned}
$$

Which divides the phase plane into two parts. Inside the boundary there is a bunch of close trajectories around point O, and Eq. (32) has period solutions. Out- side the boundary there is a bunch of open trajectories, Eq. (32) has no period solution. Whichever form the curve is, we can identify the parameters of a nonlinear system by using phase trajectory.

\section{Conclusion}

The analytical solution of the strongly nonlinear system can generally not be found, but the phase trajectory equation of some systems may be obtained. Making use of the phase trajectory equation, we identify parameters of strongly nonlinear systems in phase plane. If the phase trajectory equation is not found, there is not a mathematical model and this method can not be applied the system. This approach is applicable to both conservative systems and non-conservative system. The value ranges of the parameters of the system are not limited.

\section{References}

[1] Y.K. Cheung, S.H. Chen and S.L. Lan, A modified LindstedtPoincare method for certain strongly non-linear oscillators, Int. J. Non-Linear Mech. 26 (1991), 367-378.

[2] L. Li, Stroboscopic method for strongly nonlinear system, Acta Mechanica Sinica 22 (1990), 402-412, (in Chinese).

[3] S.B. Yuste and J.D. Bejarano, Extension and improvement to the Krylov-Bogoliubov methods using elliptic function, Int. J. Control 49 (1989), 1127-1141.

[4] J. Tang and Q. Huo, A method of identification of nonlinear system parameters from free response, Journal of Vibration Engineering 2 (1989), 62-66, (in Chinese).

[5] J. Tang, A method for identifying nonlinear system parameters in frequency Domain, Journal of Vibration and Shock 10 (1991), 67-71, (in Chinese).

[6] A.H. Nayfeh, Parametric identification of nonlinear systems, Computer \& Structures 20 (1988), 487-493.

[7] K. Yasuda et al., Experimental identification technique of vibrating structures with geometrical nonlinearities, ASME J. Appl. Mech 64 (1997), 275-280.

[8] K. Yasuda and K. Kamiya, Experimental identification technique of nonlinear beams in time domain, Nonlinear Dynamicy 18 (1999), 185-202. 

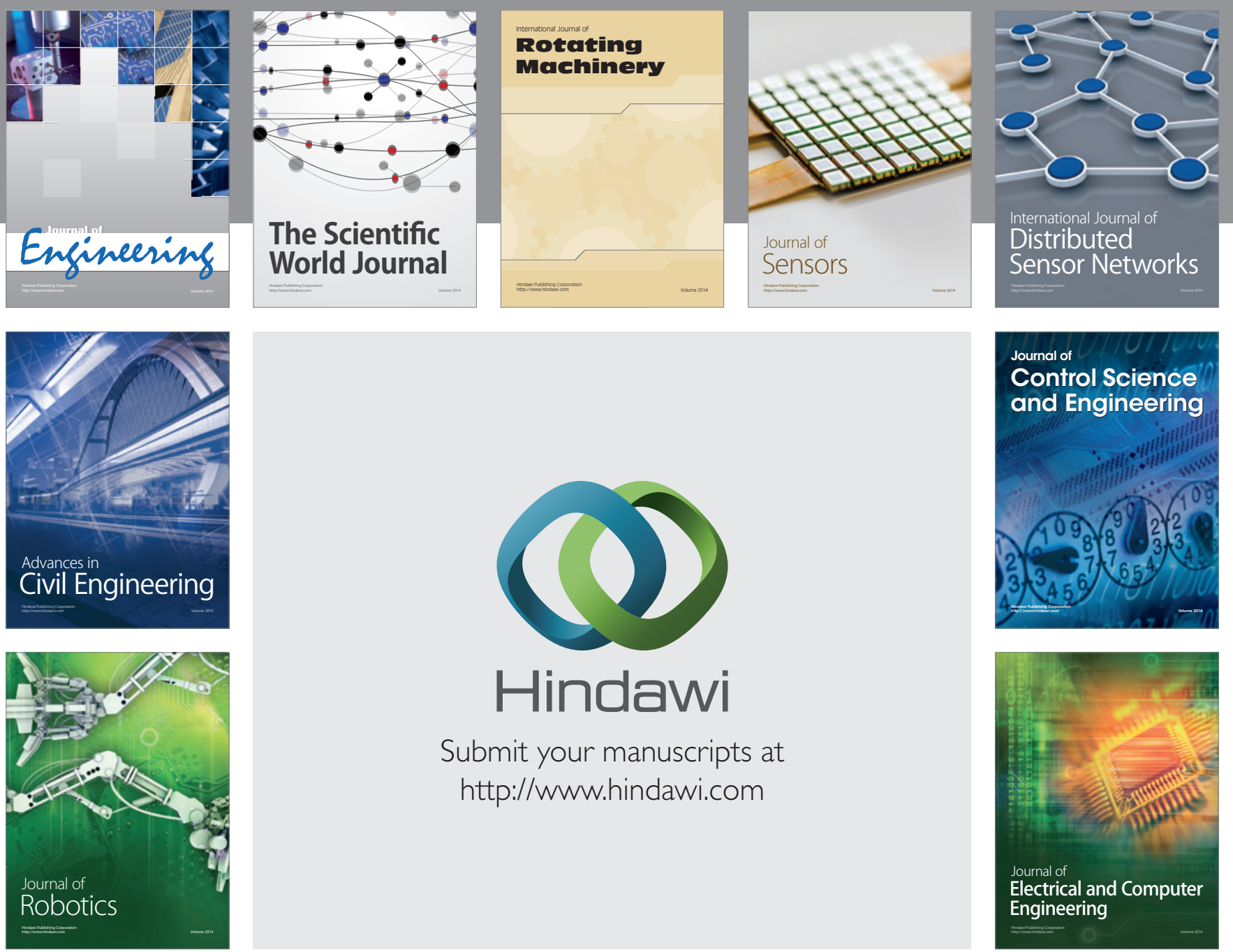

Submit your manuscripts at

http://www.hindawi.com
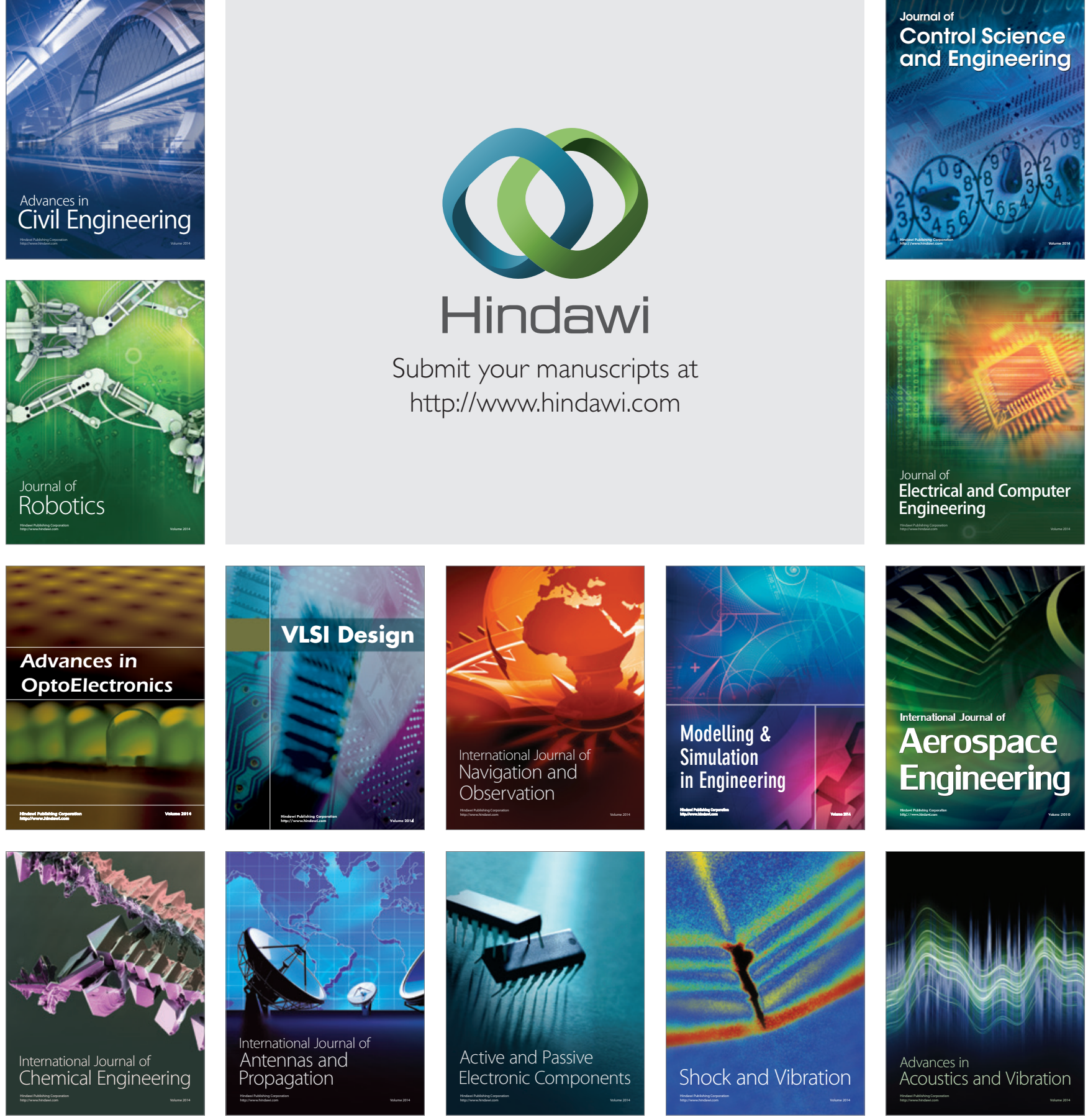